\title{
Eaux en Chine - Protection de cette ressource précieuse
}

\author{
Water in China - The protection of this precious resource
}

\author{
par Dang Weihong \\ Information Research Institute, Ministry of Water Resources, P. R. China
}

The history of water management in China is a long way to remedy the inhomogeneous repartition of water in the country. Since about ten years the rapid growth of economy and urbanization has sent a new challenge concerning the water management. The soil erosion worsening and the natural reservoirs exploiting have increased the necessity of the battle against the floods. In the towns, the water demand growth is exceeding the capacity increase of drinking water plants. In much areas of the country the water pollution is a crucial problem. Moreover the investment and the water management reform has an influence on the economic global development.

The global objective of China is to reach the average standard of living of the industrialized countries. Nowadays the water problems in China are : the floods in the South, the water shortage in the North and the pollution. These problems are considered as an obstacle to sustainable development. It is therefore necessary to take measures in order to guarantee a sufficient water supplying.

\section{I $\square$ CAUSES DE LA PÉNURIE DES RESSOURCES EN EAU}

La Chine est un pays pauvre en eau. Elle se place à l'avant-dernier rang (avant l'Inde) des grands pays pour la quantité d'eau moyenne par personne, qui est moins du tiers de la quantité moyenne du monde (voir tableau 1). La quantité d'eau moyenne par personne au nord de la Chine n'est qu'un cinquième de celle du sud de la Chine.

La Chine se divise en 9 grands bassins hydrologiques selon la limite des bassins versants des principaux fleuves (voir tableau 2). Le sud de la Chine possède plus de $81 \%$ des ressources en eau, mais regroupe seulement $55 \%$ de la population, à l'inverse du nord, où les $14 \%$ des ressources en eau font vivre $43 \%$ de la population totale. La quantité d'eau moyenne par personne au nord est de $750 \mathrm{~m}^{3}$, ce qui équivaut à $10 \%$ du niveau moyen mondial. Dans le bassin versant de Hebei qui couvre la province de Hebei, les villes de Beijing, de Tianjin, et une partie des provinces de Shanxi, Henan, Shandong, Jiangsu et Anhui, le problème d'eau est plus évident. En fait, la surexploitation des eaux souterraines des régions de ce bassin versant est de plus de $30 \%$, ce qui explique que le niveau d'eau souterraine s'est abaissé de 100 à $300 \mathrm{~m}$. Le manque d'eau naturelle au nord est la raison principale de la construction des ouvrages de transfert d'eau du sud vers le nord.

Les précipitations, spatialement non homogènes, aggravent le manque d'eau et provoquent des inondations ou des sécheresses des grands bassins versants. Par exemple, dans le bassin versant de Haihe, $70 \%-80 \%$ des précipitations se répartissent entre juillet et août, ce qui exige une capacité suffisante des réseaux d'eau pour garantir l'approvisionnement.

\subsection{La demande et la prévision}

En 1980, l'irrigation est la principale utilisation des eaux douces en Chine (82\%), viennent ensuite l'industrie (10\%), les activités agricoles (les usages domestiques, les usages pour le bétail et les activités non agricoles, $6 \%$ ) et l'usage urbain $(12 \%)$. L'utilisation de l'eau est différente dans chaque région. Le pourcentage de l'utilisation d'eau pour l'irrigation dans les régions agricoles du nord-ouest est de près de $95 \%$, mais dans les régions plus industrialisées au sud-est n'est que de $69 \%$. Dans les quinze dernières années, la demande en eau non destinée à l'irrigation a rapidement augmenté. Par exemple, de 1980 à 1987 , le pourcentage d'eau destinée à l'irrigation pour la totalité du bassin versant de Haihe est passé de $81 \%$ à $74 \%$ (SPC,1995).

En 2000, la demande prévisionnelle d'eau totale est de 765 milliards $\mathrm{m}^{3}(+72 \%$ par rapport à 1980$)$. L'augmentation de l'usage d'eau hors irrigation peut atteindre $56 \%$ de l'augmentation de l'usage d'eau totale ; ce sont les utilisations industrielles et urbaines qui augmentent le plus vite (voir tableau 3). L'augmentation de l'usage d'eau au sud sera de $62 \%$ de l'augmentation totale, au nord elle sera de $35 \%$, dans les bassins internes, de $4 \%$. 
Tableau 1. - La ressource de l'eau en Chine est limitée, surtout au nord (1995).

\begin{tabular}{|c|c|c|c|}
\hline Pays & $\begin{array}{c}\text { Quantité d'eau } \\
\text { moyenne annuelle } \\
\text { renouvelable (milliards } \mathbf{m}^{3} \text { ) }\end{array}$ & $\begin{array}{l}\text { Quantité d'eau } \\
\text { par unité de } \\
\text { surface }\left(\mathrm{m}^{3} / \mathrm{km}^{2}\right)\end{array}$ & $\begin{array}{l}\text { Quantité d'eau moyenne } \\
\text { par personne }\left(\mathbf{m}^{3}\right)\end{array}$ \\
\hline Canada & 2901 & 290944 & 98462 \\
\hline Brésil & 6950 & 816494 & 42957 \\
\hline Russie & 4498 & 263426 & 30599 \\
\hline USA & 2478 & 264659 & 9413 \\
\hline Chine & 2812 & 292917 & 2292 \\
\hline Nord de Chine & 405 & 150528 & 750 \\
\hline Sud de Chine & 2278 & 654532 & 3440 \\
\hline Inde & 2085 & 700840 & 2228 \\
\hline Monde entier & 41022 & 301988 & 7176 \\
\hline
\end{tabular}

Tableau 2. - Les grands bassins hydrologiques en Chine et leurs caractéristiques.

\begin{tabular}{|l|c|c|c|c|}
\hline Région & $\begin{array}{c}\text { Pourcentage de la } \\
\text { ressource en eau } \\
(\boldsymbol{\%})\end{array}$ & $\begin{array}{c}\text { Quantité d'eau } \\
\text { moyenne par } \\
\text { personne } \mathbf{( m}^{\mathbf{3}}\end{array}$ & $\begin{array}{c}\text { Surface des terres } \\
\text { cultivées } \\
(\boldsymbol{\%})\end{array}$ & $\begin{array}{c}\text { Population } \\
(\boldsymbol{\%})\end{array}$ \\
\hline Chine total & 100,0 & 2292 & 100,0 & 100,0 \\
\hline Nord-est & 6,9 & 1625 & 19,8 & 9,8 \\
\hline Haihe & 1,5 & 355 & 10,9 & 9,8 \\
\hline Huaihe/shandong & 3,4 & 515 & 14,9 & 15,4 \\
\hline Rivière jaune & 2,6 & 749 & 12,7 & 8,2 \\
\hline Yangtsé & 34,2 & 2280 & 24,0 & 34,8 \\
\hline Sud & 16,8 & 3534 & 6,8 & 11,0 \\
\hline Sud-est & 9,2 & 2892 & 3,4 & 7,4 \\
\hline Sud-ouest & 20,8 & 32216 & 1,7 & 1,5 \\
\hline Rivière interne & 4,6 & 5126 & 5,8 & 2,1 \\
\hline & \multicolumn{5}{|l}{} \\
\hline
\end{tabular}

Selon les estimations, l'approvisionnement maximum en eau douce en Chine pourra atteindre près de 1100 milliards de $\mathrm{m}^{3}$. A l'échelle du pays, cela peut équilibrer la demande, mais il faudrait réaliser d'immenses investissements dans des installations de distribution d'eau, de collecte des eaux usées, de traitement et de protection des sols et des eaux, sinon, le manque d'eau et les pollutions vont empêcher le développement économique et porter préjudice à la qualité de la vie du peuple.

\subsection{Principales causes de la pénurie des ressources} en eau

Le manque d'eau est un problème à long terme en Chine. Au début de l'année 80 , en prenant comme hypothèse que la précipitation annuelle est normale, le manque d'eau global en Chine est de près de 39 milliards de $\mathrm{m}^{3}$ (Wu, 1989). En 1996, on estime que le manque d'eau moyen est de près de 60 milliards de $\mathrm{m}^{3}$ (Chen, 1996). Le problème du manque d'eau de plus en plus grave est non seulement dû à l'approvisionnement mais aussi à la gestion de la demande.

Dans une grande partie de la région du nord, l'exploitation de l'eau est proche ou dépasse le niveau d'usage durable de la ressource en eau. L'insuffisance des investissements dans des installations de distribution a provoqué le manque d'eau dans des villes et des régions riches en eau. L'aggravation de la pollution fait que les ressources en eaux naturelles ne sont plus sûres et cela provoque des travaux de transfert d'eau coûteux et perturbant l'approvisionnement normal. Selon les statistiques, parmi les 270 villes qui manquent gravement d'eau, 136 villes ont un problème d'insuffisance de l'approvisionnement, 110 villes manquent d'infrastructures d'approvisionnement, 75 villes sont confrontées à des pollutions de la ressources en eau (SPC, 1995).

L'accroissement rapide de la demande en eau n'est pas une bonne chose, à cause du manque d'efficacité de la distribution et de l'utilisation de l'eau. Comme pour les principaux consommateurs, le gaspillage d'eau dans l'agriculture par l'irrigation en canaux traditionnels est près de $60 \%$. Dans des régions pauvres en eau du nord de la Chine, l'extension de la culture du riz a aggravé la crise d'eau (Lu, 1993). En outre, la grande majorité des entreprises, qui utilisent directement l'eau des rivières ou de la nappe souterraine, n'ont pas accepté l'utilisation de la recirculation de l'eau. L'efficacité moyenne des cycles industriels n'est que de $40 \%$, mais celle 
Tableau 3. - La demande croissante en eau

\begin{tabular}{|l|c|c|c|c|c|c|c|c|c|c|}
\hline & \multicolumn{2}{|c|}{ Vie urbaine } & \multicolumn{2}{c|}{ Industrie } & \multicolumn{2}{c|}{ Irrigation } & \multicolumn{2}{c|}{ Campagne } & \multicolumn{2}{c|}{ Total } \\
\hline & $\mathbf{1 9 8 0}$ & $\mathbf{2 0 0 0}$ & $\mathbf{1 9 8 0}$ & $\mathbf{2 0 0 0}$ & $\mathbf{1 9 8 0}$ & $\mathbf{2 0 0 0}$ & $\mathbf{1 9 8 0}$ & $\mathbf{2 0 0 0}$ & $\mathbf{1 9 8 0}$ & $\mathbf{2 0 0 0}$ \\
\hline Chine totale & 6,8 & 29,4 & 45,7 & 177,3 & 365,6 & 506,4 & 25,6 & 51,7 & 443,7 & 764,8 \\
\hline Nord & 3,2 & 11,1 & 17,9 & 69,3 & 13,2 & 171,7 & 9,5 & 22,0 & 162,8 & 274,1 \\
\hline $\begin{array}{l}\text { Bassin versant } \\
\text { de Haihe }\end{array}$ & 1,1 & 3,0 & 4,9 & 19,0 & 30,9 & 36,3 & 1,5 & 3,7 & 38,4 & 61,9 \\
\hline Sud & 3,4 & 17,8 & 27,1 & 105,3 & 180,2 & 272,4 & 14,4 & 27,1 & 225,1 & 422,6 \\
\hline $\begin{array}{l}\text { Rivières } \\
\text { internes }\end{array}$ & 0,2 & 0,5 & 0,7 & 2,7 & 53,2 & 62,4 & 1,7 & 2,6 & 55,8 & 68,1 \\
\hline \multicolumn{19}{|c|}{} & & \multicolumn{8}{|c|}{ Sources: Wu, 1989 et les fonctionnaires de la banque mondiale } \\
\hline
\end{tabular}

des pays développés est de $70 \%$. Le manque d'avancement de la technologie a aussi provoqué une consommation excessive ; par exemple, si on prend le charbon comme matière première, la consommation d'eau pour produire un tonne d'ammoniac est de 500 à $1000 \mathrm{t}$, si on prend le gaz naturel comme matière première, elle n'est que de $12 \mathrm{t}$. La consommation d'eau de la papeterie est de 400 à $500 \mathrm{t}$ pour produire un tonne, mais dans les pays développés, cette consommation n'est que 5 à $200 \mathrm{t}$ ( NEPA, 1997 ).

Pour équilibrer les incompatibilités de l'offre et de la demande, il faut avoir une politique rationnelle du prix de l'eau qui tienne compte des investissements, une bonne gestion et une réforme de l'organisation.

\section{II $\square$ ASPECT QUALITATIF : REJETS DE MATIÈRES POLLUANTES ET POLLU- TION DE L'EAU}

La qualité de l'eau est déterminée essentiellement par la quantité de rejets de matières polluantes. La majorité des ressources en eau de la Chine subissent le risque de cette pollution de plus en plus fortement.

\subsection{Rejets des matières polluantes}

En Chine, les matières polluantes de l'eau proviennent principalement des eaux usées industrielles et urbaines, des flux pollués des engrais chimiques, des pesticides et des rejets fécaux des animaux, ainsi que des lessivages des déchets solides.

En 1995, les rejets des matières polluantes industrielles et urbaines vers le milieu naturel furent respectivement de 27 milliards de tonnes et de 15 milliards de tonnes. Bien que depuis 1990, les rejets des eaux usées des entreprises industrielles, là où il existe un contrôle, se soient stabilisés, les rejets en eaux polluées des petites entreprises rurales et urbaines ont augmenté rapidement. Cela demande donc des mesures urgentes en vue du contrôle des rejets de polluants de celles-ci.

$\mathrm{Au}$ nord de la Chine, dans des régions qui manquent d'eau, la pollution de l'eau est plus grave, car la capacité de l'autoépuration (dilution) est limitée. En 1995, les rejets des eaux usées au nord et au sud furent respectivement de $4 \%$ et $1 \%$ des ruissellements de surface. Dans le bassin versant de Haihe, la proportion des eaux polluées et des ruissellements est de $11 \%$. Bref, la pollution de l'eau au nord est plus grave qu'au sud.
En 1995, dans les entreprises où il existe un contrôle de la pollution, la DCO totale des rejets des entreprises de papeterie, de l'agro-alimentaire et des industries chimiques sont respectivement de $42 \%, 28 \%$ et $9 \%$. Les industries chimiques, sidérurgiques et mécaniques rejettent beaucoup de dérivés du pétrole : respectivement $29 \%, 21 \%$ et $13 \%$. L'industrie chimique rejette du mercure, de l'arsenic, du cyanure et du phénol volatile. Les quantités de rejets pour chaque matière sont respectivement $42 \%, 46 \%, 42 \%$ et $28 \%$.

En raison de l'utilisation universelle des engrais chimiques et des pesticides et de l'augmentation rapide de la production de bétail, la pollution de l'eau par les activités agricoles est de plus en plus grave. Dans les dix années passées, la quantité d'engrais chimiques et pesticides utilisée a doublé. En 1995, les quantités utilisées sont respectivement de 36 millions de tonnes et 1 million de tonnes.

Les déchets solides, surtout les lessivages des déchets toxiques, peuvent provoquer la détérioration de la qualité de l'eau dans une grande partie du pays. L'accumulation des déchets solides en Chine a déjà fort augmenté, en particulier celle des déchets industriels qui ont dépassé 6,6 milliards de tonnes, et s'accroît chaque année de 0,18 milliards de tonnes, parmi lesquels, 10\% sont toxiques et nuisibles. En 1995, la quantité totale des rejets des déchets solides dans l'eau par les entreprises industrielles qui sont sous contrôle de pollution fut de 6,5 millions de tonnes ( NEPA,1996 ).

Les fuites et les débordements d'essence ou diesel des bateaux et des navires ont pollué les rivières, les lacs et les côtes. Les 30000 navires qui passent par le Yangtsé déversent chaque année plus de 5000 tonnes d'essence ou diesel et échappent à tout contrôle. Selon les statistiques, la quantité totale de rejets des eaux usées par ces navires est près de 90 millions de tonnes par an, la quantité totale de rejets des déchets solides est de près de 80000 tonnes par an ( MOT, 1997 ).

\subsection{La qualité de l'eau}

En Chine, on distingue 5 classes de qualité d'eau. Les trois premières permettent la baignade et l'usage comme ressource de l'eau potable. La quatrième classe est limitée à l'usage industriel ou loisir sans baignade. La cinquième classe ne convient qu'à l'irrigation. L'Etat a fixé pour chaque classe la valeur-guide de $\mathrm{pH}$ et la concentration maximum en 28 principales matières polluantes; si l'une des matières polluantes a dépassé le seuil, l'eau ne peut pas être placée dans cette classe. 
Tableau 4.-De nombreuses rivières urbaines n'arrivaient pas à atteindre la classe 5 en 1995.

\begin{tabular}{|l|c|c|c|c|c|}
\hline \multicolumn{1}{|c|}{$\%$} & classe 2 & classe 3 & classe 4 & classe 5 & hors classe \\
\hline Au nord (66)* & 4,6 & 3,0 & 18,2 & 22,7 & 51,5 \\
\hline Au sud (69)* & 17,4 & 18,8 & 29,0 & 8,7 & 26,1 \\
\hline Total (135)* & 11,1 & 11,1 & 23,7 & 15,6 & 38,5 \\
\hline * nombre de rivières & \multicolumn{4}{|c|}{ Sources: NEPA, 1997 } \\
\hline
\end{tabular}

On considère les eaux urbaines comme les plus polluées à cause du rejet d'une grande quantité d'eaux usées domestiques et industrielles sans traitement. Selon l'inspection sur 135 rivières urbaines, il y a 34 des 66 rivières urbaines du nord et 18 des 69 rivières urbaines du sud qui ont dépassé la cinquième classe de la qualité de l'eau et sont presque comme des égouts. Aucune rivière sous inspection n'a atteint la première classe. (Voir tableau 4).

La pollution des rivières urbaines est principalement due à des matières organiques, surtout des matières pétrolières et phénols volatiles. Les autres pollutions sont les matières organiques, azote ammoniacal et mercure (voir tableau 5). Entre 1991 et 1995, la quantité de rejets des principales matières polluantes est relativement stabilisée ou abaissée légèrement, mais la pollution d'azote ammoniacal augmente de près de $50 \%$ à cause de l'accroissement des rejets des eaux usées urbaines sans traitement, des effluents agricoles contenant des engrais azotés et des eaux usées résiduelles des usines d'engrais chimiques.

La pollution des rivières du nord de la Chine est plus grave que celle du sud. La qualité des eaux du bassin versant de Haihe et de $50 \%$ du bassin versant de Luanhe ne peut pas atteindre la cinquième classe. Selon l'examen des échantillons, $94 \%$ des rivières de Liaohe ne conviennent pas à la production d'eau potable. En 1995, la pollution industrielle du bassin versant de Huaihe fut un désastre, ce qui a obligé le gouvernement central à la mise en place de mesures d'urgence pour contrôler les rejets d'eaux usées

Les lacs et les réservoirs présentent généralement un risque d'eutrophisation à cause de la croissance des teneurs en azote et phosphates. Dans les grands lacs d'eau douce, l'eutrophisation des lacs Taihu, Chaohu, et Dianchi est la plus grave. Les teneurs en azote de ces trois grands lacs sont respectivement $2,5 \mathrm{mg} / \mathrm{l}, 3,8 \mathrm{mg} / \mathrm{l}$, et $2,3 \mathrm{mg} / \mathrm{l}$ et ont toutes dépassé le seuil 2,0 mg/l de la cinquième classe. La teneur en mercure de ces lacs est aussi élevée, la teneur moyenne de mercure sur cinq ans des lacs de Chaohe et Dianchi a dépassé le niveau de $0,0001 \mathrm{mg} / \mathrm{l}$ de la troisième classe (NEPA, 1996).

La qualité de l'eau souterraine est abaissée aussi, surtout dans les régions du nord. La ressource de l'eau souterraine est utilisée largement pour compenser le manque d'eau superficielle. Le niveau de la dureté, de la minéralisation et de la concentration de nitrate a augmenté. Les matières polluantes comme le phénol volatil, le cyanure et les nitrates sont toutes apparues dans les eaux souterraines, ce qui explique les impacts des infiltrations des eaux usées superficielles.

La qualité de l'eau de mer est également dégradée, la pollution d'azotes inorganiques est plus grave et plus étendue. Dans les 839 échantillons contrôlés, la moitié a dépassé la valeur maximum. La pollution la plus grave se trouve à l'entrée de la mer, dans le golfe, le port et les abords des grandes ou moyennes villes ; par exemple, à l'estuaire du Yangtsé, dans le golfe de Hangzhou et les régions maritimes de la pêcherie de Zhoushan, la teneur en azote, phosphate inorganique a dépassé la limite maximum respective de $100 \%$ et $85 \%$ (NEPA, 1986).

En somme, la capacité d'autoépuration de la plupart des rivières de la Chine est épuisée par la pollution de plus en plus grave. Depuis 1980, la qualité de l'eau des principales rivières s'est détériorée : une grande partie est tombée dans la troisième, la quatrième ou la cinquième classe.

Comme la Chine a réussi à fournir de l'eau potable à la population, les épidémies causées par la pollution de l'eau sont bien contrôlées. Mais si on ne renforce pas les mesures de contrôle de la pollution, la qualité des rivières, des lacs, des eaux souterraines et des régions maritimes sera davan-

Tableau 5. - Principales matières polluantes des rivières urbaines en 1995.

\begin{tabular}{|l|c|c|}
\hline Matières polluantes & classe $\mathbf{3}(\mathbf{m g} / \mathbf{l})$ & Taux de densité moyen annuel dépassant classe 3 $(\boldsymbol{\%})$ \\
\hline Matière pétrolières & 0,05 & 70,5 \\
\hline $\mathrm{K}_{2} \mathrm{MnO}_{4}$ & 6 & 51,1 \\
\hline $\mathrm{DBO5}$ & 4 & 44,7 \\
\hline Azote ammoniacal & 1 & 44,4 \\
\hline Phénol volatil & 0,005 & 37,3 \\
\hline $\mathrm{O}_{2}$ dissous & 5 & 31,9 \\
\hline $\mathrm{Hg}$ total & 0,0001 & 18,8 \\
\hline $\mathrm{Cd}$ total & 0,005 & 7,7 \\
\hline $\mathrm{Pb}$ total & 0,05 & 7,0 \\
\hline \multicolumn{2}{|l}{} \\
\hline
\end{tabular}


tage détériorée. Les impacts potentiels seront plus graves.

Maintenant le gouvernement chinois a déjà pris conscience des graves conséquences de la pollution de l'eau. Depuis la fin de 1980 , on a eu une remarquable amélioration du contrôle de la pollution. Ces dernières années, la pollution des grandes rivières s'est stabilisée. En 1995, la Chine a commencé la mise en œuvre à grande échelle du programme de contrôle de la pollution du bassin versant de Huaihe ; les autres parties du programme sont en cours d'exécution ou planifiées. La Chine a l'espoir d'inverser la tendance concernant la pollution au début du XXIe siècle.

\section{III — GESTION DES EAUX ET LOIS CONCERNANT LE CONTRÔLE DE LA POLLUTION (SITUATION EXISTANTE)}

Les organismes de gestion des eaux et du contrôle de la pollution en Chine se divisent en deux échelles : Etat et région. Les lois (loi sur l'eau de la République Populaire de Chine; Loi de Prévention et de Traitement de la Pollution des Eaux de la République Populaire de Chine) ont déterminé les fonctions de chaque niveau des organismes de l'administration et présentent une base des règles du régime de la gestion des ressources et de l'environnement des eaux de la Chine.

\subsection{Organismes d'Etat}

Le ministère des Eaux se charge de la gestion des eaux du pays entier, y compris : l'aménagement et la protection des grands bassins versants ; la planification de l'approvisionnement en eau des grandes villes; la construction des installations de base de l'irrigation ; l'exécution des projets de protection des sols et des eaux ; la construction des ouvrages hydrauliques ; la construction et la gestion des grands et moyens réservoirs pour la lutte contre les inondations, l'irrigation, l'approvisionnement en eau et les travaux hydrauliques. Les sept comités des grands bassins versants subordonnés au ministère des Eaux se chargent de la coordination de la gestion des eaux au niveau du bassin versant. Dans chaque province, ville et autorité de district, il y a un bureau de l'eau relativement indépendant.

Le bureau de la Protection de l'Environnement d'Etat a pour mission d'établir des normes et des règles du contrôle de la pollution de l'eau, de protéger la qualité de l'eau du pays entier. L'exécution des normes et des règles fait partie de la responsabilité des bureaux locaux de la protection de l'environnement.

Le ministère de la Construction et ses organismes locaux correspondants se chargent essentiellement de la conception et de la construction des systèmes de l'approvisionnement en eau urbaine et des systèmes de collecte et de traitement des eaux usées urbaines.

Le ministère de l'Industrie de l'Energie électrique est aussi un organisme important de gestion des ressources en eau par ses grands systèmes hydroélectriques ; comme les autres organismes ministériels, il fait appel à la coordination des organismes régionaux ou locaux correspondants.

Le ministère des Communications se charge des aménagements et de la gestion des activités de transport dans les rivières internes ou littorales. Le ministère de la Géologie et des Ressources minières se charge d'explorer des ressources en eaux souterraines. Le ministère de la Santé Publique se charge d'inspecter la qualité de l'eau potable et les maladies associées à l'eau contaminée.

La commission de la Coordination de l'Eau d'Etat a pour tâche de renforcer la gestion intégrée des eaux, les échanges d'informations et les coordinations des secteurs. Elle est dirigée par le ministère des Eaux ; ses membres sont les représentants des ministères concernés, du Bureau de la Protection de l'Environnement d'Etat, de la Commission du Plan d'Etat et de l'Académie des Sciences. Quelques provinces, villes et départements autonomes ont des commissions de la coordination des eaux correspondants.

\subsection{Gestion des bassins versants}

Le comité de la gestion du bassin versant est un organisme important de l'administration, de la coordination et de la consultation des ressources en eau à l'intérieur de son propre bassin versant. Ses fonctions importantes sont la prévention et la lutte contre les inondations, les transports solides et les sécheresses. Dans les bassins versants qui manquent de ressource en eau, ce comité se charge souvent de résoudre les conflits interprovinciaux de cette ressource ; il surveille aussi la qualité de l'eau en collaboration avec les ministères concernés et Les bureaux de la protection de l'environnement. Chaque comité du bassin versant a un personnel suffisant (par exemple, au comité du bassin versant de la rivière jaune, il y a 29000 employés).

Les bureaux des Eaux provinciaux se chargent de la planification, de l'étude, de la conception, de la construction, de l'exploitation et de la gestion des ouvrages d'irrigation et de drainage, des ouvrages de protection contre les inondations et des ouvrages hydroélectriques ruraux. Les bureaux des Eaux de bourgs ou de districts se chargent directement des constructions et de l'entretien des ouvrages d'irrigation, des ouvrages de protection contre les inondations et des réservoirs de moyenne dimension de la plupart des sections des rivières. Les bureaux des eaux de communes et de villages se chargent des constructions et de l'entretien des ouvrages auxiliaires et des réservoirs de petite dimension des affluents. Les employés des bureaux des Eaux de bourgs ou de districts se divisent en groupes selon les parcelles de l'irrigation, ont pour mission l'exploitation et l'entretien des ouvrages, la planification et distribution de l'eau et le recouvrement des factures. Aux bureaux des eaux de communes et de villages, chaque groupe sélectionne ses représentants pour établir une commission des rivières et canaux ; ce genre de commission indiqué par des employés des régions de l'irrigation, se charge des gestions sur place de l'usage d'eau agricole.

Les organismes de la protection de l'environnement des provinces, des villes et des districts prennent la responsabilité du contrôle de la pollution de l'eau. Chaque bureau de protection de l'environnement se charge d'exécuter des règles du contrôle de la pollution de l'eau d'Etat ou locaux. Mais les problèmes de la pollution de l'eau des régions aux frontières du bassin versant ne sont pas encore résolus. La qualité de l'eau des régions frontières fait partie de la responsabilité des organismes de la protection de l'eau du comité du bassin versant, mais ils manquent souvent de fonds et d'autorités. Le bassin versant de Huaihe est un essai prioritaire du contrôle de la pollution de l'eau au niveau du bassin versant de Chine. 
Exemple: Règles provisoires du contrôle de la pollution de l'eau du bassin versant de Huaihe

La gestion du contrôle de la pollution de l'eau du bassin versant de Huaihe date de 1995. L'Etat a mis sur pied une équipe de direction conjointe dirigée par le ministère des Eaux et le bureau de la Protection de l'environnement d'Etat. Ses membres comprennent des représentants des quatre provinces du bassin versant de Huaihe et les ministères concernés. Un plan du contrôle de la pollution du bassin versant entier a été établi et des règles provisoires du contrôle de la pollution de l'eau sont adoptées. Le gouvernement chinois fait du contrôle de la pollution de l'eau du bassin versant de Huaihe un exemple pour les autres bassins versants. Il est manifeste que les principes du contrôle de la pollution de l'eau en Chine ont progressé d'un grand pas:

- le contrôle de la pollution de l'eau est effectué pour la première fois à l'échelle du bassin versant et il est géré conjointement par le ministère des Eaux et les secteurs de la protection de l'environnement.

- la charge des pollutions est déterminée par les provinces, les villes et les districts de chaque section du bassin versant.

- les entreprises qui créent trop de pollutions ont pour objectif la diminution de la charge polluante ; les entreprises qui sont de gros pollueurs et qui ne sont pas rentables doivent être fermées.

- l'exécution du contrôle au terme limité pour les entreprises polluantes.

- les gouvernements locaux doivent incorporer le plan de diminution de la charge polluante au plan de développement économique.

- les amendes et les taxes de la pollution doivent être affectées directement aux installations de contrôle de la pollution.

- établir des systèmes d'inspection pour vérifier les données de la charge polluante fournies par les entreprises.

- l'inspection de la qualité de l'eau dans les régions frontalières fait partie de la responsabilité du comité du bassin versant de Huaihe ; l'équipe de direction doit être informée des données d'inspection de la qualité de l'eau.

Quel que soit le contrôle de la pollution de l'eau du bassin versant de Huaihe, il apparaît comme un pas important pour le contrôle de la pollution de l'eau en Chine, mais ses plans susmentionnés auront rencontré beaucoup de difficultés pour perdurer :

- les règles provisoires du contrôle de la pollution de l'eau ne prévoient pas les financements pour la diminution de la pollution. Les dépenses pour fermer des entreprises sont à la charge des gouvernements locaux. Comme ces dépenses sont assez importantes, les gouvernements central et provincial doivent accorder des aides financières.

- les règles provisoires du contrôle de la pollution de l'eau ne prévoient pas les moyens concrets de diminution de la pollution des entreprises. Les entreprises industrielles, surtout celles des villages, manquent généralement des technologies du contrôle de la pollution. Il faut donner aux communes et aux villages des aides techniques pour les aider à établir et à exécuter les projets de la diminution de la pollution efficace.

- les règles provisoires n'ont pas proposé de traitements communs aux eaux usées domestiques et industrielles afin de diminuer le prix de revient de l'unité et le mettre à la portée des industriels. Chaque ville ou commune doit garantir que les eaux usées industrielles entrent dans les systèmes de traitement urbains après des prétraitements à l'intérieur de l'usine qui éliminent les matières toxiques et les métaux lourds.

- les règles provisoires n'ont pas présenté l'option de réunir les organismes de collecte et de traitement des eaux usées urbaines. Actuellement, la collecte des eaux usées est de la responsabilité de l'administration de la municipalité. Chaque district de ville doit collecter une partie des fonds. Mais le traitement des eaux usées fait partie de la responsabilité des différents secteurs.

Les bureaux de protection de l'environnement provinciaux et communaux se chargent d'inspecter la qualité de l'eau des rivières. Le comité du bassin versant se charge de contrôler la qualité de l'eau dans les régions frontalières. Cette séparation provoque une certaine confusion, car le niveau et la qualité de l'inspection de chaque secteur sont très différents.

\subsection{Approvisionnement et traitement des eaux urbaines}

Le ministère de la Construction se charge de la gestion financière des approvisionnements et des traitements des eaux urbaines. Les projets d'investissements importants doivent être approuvés par ce ministère. L'approvisionnement en eau urbain est une obligation conjointe des gouvernements locaux et des entreprises. Selon les statistiques de début 90 , l'eau captée par des entreprises pour la production et la vie des employés occupe près de la moitié de la fourniture en eau urbaine.

Depuis longtemps, l'approvisionnement en eau des villes est considéré comme un service social fondamental du gouvernement. La gestion de la demande de l'eau est une façon de fixer la quantité d'eau. Le prix de l'eau traditionnel ne représente pas la valeur des ressources en eau ni le prix de revient de la récupération. Le prix de l'eau captée directement par l'usager est encore moindre (La Banque Mondiale, 1994).

Le gouvernement a commencé l'exécution d'une série de réformes : augmenter le prix de l'eau, augmenter le prix de l'eau captée par l'utilisateur, diminuer la dépendance de la quantité d'eau fixée. Actuellement en Chine, il existe généralement des subventions pour l'approvisionnement en eau, même pour les usages commerciaux. La faiblesse des prix aboutit à une baisse d'efficacité dans l'utilisation des ressources en eau. Les entreprises acceptent une technologie qui demande beaucoup d'eau et les investissements pour la ressource en eau ne sont pas suffisants.

Fin 1980 , seulement $57 \%$ des villes collectaient les eaux usées. En 1990, étant donné le développement urbain et communal, cette situation ne s'est pas beaucoup améliorée. Le niveau de traitement des eaux usées urbaines est encore plus bas à cause de l'insuffisance des investissements. En 1992 , il n'y a que $4,5 \%$ des eaux usées urbaines qui sont traitées mais si l'on inclut les eaux usées industrielles traitées, le taux de traitement des eaux usées urbaines s'élève à $17 \%$.

Les bureaux de protection de l'environnement d'Etat et locaux se chargent de l'inspection et de la gestion du contrôle de la pollution des eaux usées industrielles. Les principales cibles sont les entreprises d'Etat, car elles sont des pollueurs de l'environnement. Le développement rapide des entreprises de villages a étendu les problèmes relativement concentrés dans les villes à un grand domaine. Beaucoup d'affluents des grandes rivières ont subi maintenant une grave pollution. 
Tableau 6. - Le prix de l'eau des villes de la province de Hebei (1996).

\begin{tabular}{|l|c|c|c|c|c|c|}
\hline $\begin{array}{l}\text { Usagers } \\
\text { yuan/m } \mathbf{m}^{\mathbf{3}}\end{array}$ & Handan & Henshui & Langfang & Qinhuangdao & $\begin{array}{c}\text { Shijia } \\
\text { zhuang }\end{array}$ & Tangshan \\
\hline Domestique & 0,50 & 0,45 & 0,40 & 0,40 & 0,65 & 0,50 \\
\hline Service & 0,50 & 0,75 & 0,80 & 0,60 & 0,60 & 0,50 \\
\hline Industrie* & 0,80 & 1,00 & 1,00 & 0,80 & 1,20 & 0,70 \\
\hline Commerce & 1,25 & - & 1,50 & 2,50 & 1,40 & 1,20 \\
\hline Moyenne & 0,63 & 0,85 & 0,76 & 0,73 & 0,90 & \\
\hline * Le prix de l'eau captée par soi-même est encore plus bas Sources: l'estimation des fonctionnaires de la banque mondiale \\
\hline
\end{tabular}

\subsection{Problèmes de la gestion des eaux et contrôle de la pollution}

Malgré le fait qu'à présent le régime de la gestion des eaux est relativement systématique, il faut améliorer les points suivants :

- il n'y a pas d'encadrement suivi des règles de la gestion des eaux aux frontières des bassins versants. Le ministère des Eaux a rédigé un programme, il faut l'appliquer le plus tôt possible.

- pour les sept grands bassins versants, il manque une gestion des eaux en fonction du temps et des saisons sauf pour ce qui est de la lutte contre les inondations. Dans les provinces qui connaissent des pénuries d'eau, il faut gérer l'eau selon le temps et les saisons.

- Il devrait y avoir une coordination entre les secteurs des sept grands bassins versants dans le domaine de l'exploitations des ouvrages hydrauliques.

- la planification des utilisations des ressources en eau demeure une préoccupation dans chaque province mais cela est défavorable aux intérêts des bassins versants. Les comités des bassins versants doivent avoir plus de compétences dans le domaine de la vérification des projets des ressources en eau de chaque province et de la garantie de l'unanimité des projets communs aux bassins versants.

- le prix de l'eau est trop bas pour satisfaire aux dépenses de l'administration des comités des bassins versants. Comme il est interdit aux comités des bassins versants de collecter des taxes pour la gestion des barrages, la lutte contre les inondations et envasements, le gouvernement doit fournir beaucoup de fonds pour aider le fonctionnement des comités des bassins versants, mais ce n'est pas cohérent à long terme.

- les comités des bassins versants ne se chargent que des gestions de la pollution des eaux dans les régions frontalières des bassins versants, ces organismes subordonnés de la protection de l'eau n'arrivent pas à inspecter toutes les régions à cause de l'insuffisance de crédits. En effet, les comités des bassins versants doivent inspecter toutes les sections des rivières, y compris leurs affluents, collecter et vérifier toutes les données de la qualité de l'eau des bureaux de la protection de l'environnement provinciaux et communaux pour avoir une connaissance complète de la qualité de l'eau des bassins versants.

Les problèmes structurels du régime de la gestion des eaux en Chine sont la séparation de la gestion des eaux et du contrôle de la pollution des eaux, la répartition des compétences entre l'Etat et les secteurs locaux. Le gouvernement est en train mettre en œuvre des mesures pour résoudre ces problèmes.

\section{5. Augmentation du prix de l'eau}

Pour garantir l'approvisionnement en eau pure et en quantité suffisante sans accroître beaucoup les dépenses du gouvernement, il est nécessaire de revoir le mode de gestion actuel et d'augmenter le prix de l'eau et la redevance de traitement des eaux usées.

Dans les régions de plaine du nord de la Chine, beaucoup de villes sont en train de diminuer la pression de l'approvisionnement en eau et le gaspillage, de gérer la demande d'eau en fixant un prix rationnel et en donnant des aides techniques aux usagers. Bien que le prix de l'eau reste encore très bas, les gouvernements centraux et locaux sont en train d'encourager l'augmentation du prix de l'eau pour qu'il puisse refléter le prix de revient de l'approvisionnement en eau et la rareté de cette ressource. Dans la ville de Taiyuan de la province de Shanxi, le bureau du prix urbain a annoncé que pendant cinq ans, le prix de l'eau va augmenter de 1,24 yuan $/ \mathrm{m}^{3}$ à 4,94 yuan $/ \mathrm{m}^{3}$ pour récompenser le prix de revient de l'approvisionnement. Dans la province de Hebei, beaucoup de villes ont déjà présenté leur manière d'augmenter le prix de l'eau pour compenser le prix de revient de l'approvisionnement en eau. Le tableau 6 montre que le prix de l'eau dans ces villes est entre 0,5 et $0,9 \mathrm{yuan} / \mathrm{m}^{3}$. Actuellement, le prix de revient marginal des nouveaux travaux d'approvisionnement en eau est de plus de $1,2 \mathrm{yuan} / \mathrm{m}^{3}$.

Dans des villes côtières, le prix de l'eau acceptable socialement est estimé à $1,8 \mathrm{yuan} / \mathrm{m}^{3}$. Dans des villes pauvres en eau, par exemple la ville de Taiyuan du bassin versant de Haihe, le prix de l'eau acceptable est de $4,3 \mathrm{yuan} / \mathrm{m}^{3}$. Le coût économique de l'eau pour les usages industriels dans la province de Shanxi et tout le bassin versant de Haihe est près de $24 \mathrm{yuan} / \mathrm{m}^{3}$. Comme la province de Shanxi est une très importante base industrielle de charbon de la Chine, l'insuffisance de l'approvisionnement en eau va empêcher son développement.

\subsection{Renforcer le traitement des eaux usées}

Si la qualité et la quantité du traitement des eaux usées urbaines et industrielles ne peuvent pas être élevées, les ressources en eau de première et de troisième classe de nombreuses villes seront de plus en plus rares. Le long des grandes rivières du nord de la Chine, on rencontre de graves problèmes de captage d'eau potable. Les ouvrages de transfert d'eau de grande dimension coûtent très cher et durent longtemps. Dans des villes ou des communes où l'approvisionnement de l'eau reste insuffisant à cause de la pollution, le traitement des eaux usées peut apporter non seulement des avantages au plan sanitaire, mais aussi une force productive industrielle et agricole considérable. Si le traitement des 
eaux usées reste toujours en retard, l'augmentation de l'approvisionnement en eau et de la consommation peuvent provoquer encore plus de déversements d'eaux usées.

Selon l'analyse de la Banque Mondiale, le coût du traitement primaire des eaux usées urbaines (éliminer $30 \%$ de DBO5 et la plupart des métaux lourds) est de 0,5 yuan $/ \mathrm{m}^{3}$ (prix de 1994), le coût du traitement secondaire (éliminer $85 \% \sim 95 \%$ de DBO5) doit augmenter à 0,77 yuan $/ \mathrm{m}^{3}$. Si le prix de revient du traitement est partagé par chaque usager, il doit augmenter de $50 \%$ ou encore plus. Pour la plupart des usagers, ce coût est acceptable. L'augmentation de la taxe pour le traitement des eaux usées peut fournir les fonds nécessaires à l'agrandissement des ouvrages de traitement des eaux usées urbaines. Dans des villes qui manquent d'eau, les eaux usées traitées peuvent fournir des ressources en eau moins chères pour les industriels.

Comme l'irrigation agricole est la principale activité d'utilisation des eaux usées, les eaux usées non traitées et déversées directement dans les rivières et les lacs vont entraîner de graves conséquences pour l'agriculture. Normalement, on ne peut pas utiliser des eaux de qualité inférieure à la classe 5 pour l'irrigation agricole, car ces eaux contiennent beaucoup de métaux lourds et des matières toxiques. En 1993, $8 \%$ des terres irrigables ont utilisé des eaux usées moins bonnes que de classe 5 , provoquant une diminution de la production agricole de près de 10 milliards tonnes (NEPA, 1997). Maintenant, le traitement des eaux usées urbaines en Chine est loin d'être suffisant pour atteindre la classe 5. On dépasse la critère de DCO5 de plus de 13 fois, et si le niveau de la collecte et du traitement des eaux usées urbaines et industrielles n'augmente pas, la production agricole de la Chine va diminuer encore davantage.

L'impact futur des eaux usées sur la production agricole de la Chine peut être estimé par le calcul du modèle de la demande des neuf grandes rivières. En supposant qu'en l'an 2000 , la proportion de la quantité du traitement des eaux usées augmente de $20 \%$ à $30 \%$ de la quantité du déversement total, la variation du taux du traitement du modèle va augmenter de $50 \%$ à $75 \%$; si ce taux augmente de $30 \%$ à $50 \%$, l'augmentation de la production agricole en l'an 2000 va atteindre 24 millions de tonnes (selon la consommation moyenne par personne en 1996, cela est suffisant pour nourrir 65 millions de personne); si ce taux augmente encore de $50 \%$ à $75 \%$, l'amplitude de l'augmentation de la production agricole sera moins grande, près de 7 millions de tonnes.

\section{IV — PRINCIPES DURABLES DE LA GES- TION DES EAUX (PROPOSITIONS)}

Maintenant la Chine est face au défi de résoudre le problème du manque d'eau chronique de la région du nord et de contrôler la pollution de l'eau du pays entier. Les mesures prédominantes sont l'augmentation des investissements de l'approvisionnement en eau et la gestion des eaux. On est en train d'augmenter le prix de la distribution de l'eau et la taxe pour le traitement des eaux usées, mais cela est loin d'être un système de prix avec une économie rationnelle et un intérêt social. L'exécution de ces mesures est le rôle des organismes de protection de l'eau d'Etat et locaux. Il faut établir un système de gestion des eaux sur la base des bassins versants et éclairer les responsabilités de chaque secteur. Cela est indispensable pour la gestion intégrée des eaux et du contrôle de la pollution.

\subsection{Expérience des pays développés dans le domaine de la gestion des eaux et du contrôle de la pollution}

La majorité des pays développés exécute une gestion intégrée des ressources en eau et du contrôle de la pollution. La Chine peut bénéficier des expériences ci-dessous :

- La gestion des eaux et le contrôle de la pollution appartiennent aux responsabilités des bassins versants, et les organismes impliqués fonctionnant comme des entreprises, les bassins versants doivent être conférés aux autorités de gestion dans les domaines suivants : les captages, la lutte contre les inondations, les drainages, les déversements d'eaux usées dans les rivières, la protection de la qualité de l'eau (établir un système d'inspection de la qualité de l'eau du bassin versant tout entier), la navigation, la pollution littorale, la gestion de la quantité d'eau, la collecte des factures, y compris le prix de l'inspection, de la lutte contre les inondations et des drainages, les taxes des rejets des eaux usées et les amendes des déversements d'eaux usées dans les rivières.

- Toutes les villes et communes doivent établir un système unitaire du traitement des eaux usées urbaines et industrielles. Les eaux usées industrielles avant leur entrée aux systèmes du traitement des eaux usées urbaines doivent avoir une prétraitement pour éliminer les matières toxiques et les métaux lourds. Il est interdit aux entreprises de déverser directement des eaux usées industrielles aux rivières naturelles.

- Il faut augmenter les prix de captage, de fourniture, du traitement des eaux usées, de lutte contre les inondations et de rejets pour la récupération du prix de revient de toutes ces activités.

\subsection{Gestion de la demande par ajustement du prix de l'eau et de la taxe sur le traitement des eaux usées}

L'augmentation du prix de l'eau a pour but de protéger la ressource en eau. Bien que ces dernières années, la Chine ait déjà procédé à plusieurs réformes du prix de l'eau et de la gestion des eaux, le prix de l'approvisionnement en eau et la taxe du traitement des eaux usées restent encore très bas, ce qui conduit à l'augmentation de la demande en eau, des pollutions graves et à l'insuffisance de l'investissement.

Le prix de l'eau doit refléter la rareté de cette ressource et le prix de revient économique de cette ressource. Le ministère des Eaux a annoncé récemment l'ouverture progressive du marché du prix de l'eau à partir des ouvrages nouveaux. Quelques gouvernements locaux ont permis aux investisseurs privés ayant construit des ouvrages de fixer le prix de l'eau librement. Le prix de l'eau économique peut renforcer la gestion de la demande et fournir une aide financière aux entreprises de fourniture d'eau pour qu'elles puissent augmenter la qualité de l'entretien et du service, et se déployer progressivement.

Pour éviter aux entreprises de surexploiter des eaux souterraines et superficielles, il faut augmenter le prix de l'eau que l'on capte soi-même jusqu'au niveau du prix de l'eau commerciale. Une exploitation rationnelle et un prix convenable pour l'approvisionnement en eau peuvent diminuer les investissements du gouvernement pour l'exploitation et l'approvisionnement en eau. Les entreprises peuvent se débarrasser des réseaux d'approvisionnement en eau d'intérêt local, et chaque ville peut redistribuer ses investissements. 
L'augmentation de la taxe sur le traitement des eaux usées peut encourager des villes à investir dans des installations du traitement des eaux usées. Pour éviter la détérioration continue de la qualité de l'eau, les villes et les communes doivent augmenter le niveau du traitement des eaux usées au rythme de l'accroissement des déversements d'eaux usées. La construction de stations du traitement des eaux usées est le seul moyen de résoudre ce problème. Les revenus courants du gouvernement ne peuvent pas satisfaire aux besoinx d'investissements du traitement des eaux usées. Chaque ville doit augmenter le prix du traitement des eaux usées domestiques, commerciales et industrielles à un niveau tel qu'elle puisse compenser le prix de revient du traitement des eaux usées. Bien que les dirigeants conservent une certaine réticence à demander aux usagers la taxe sur les eaux, les responsables des villes doivent avoir conscience que le prix de revient du traitement des eaux usées doit être compensé directement ou indirectement. Actuellement, le paiement indirect entraîne des déversements excessifs d'eaux usées, et d'un autre côté on ne peut pas rassembler les investissements pour le traitement des eaux usées, ce qui a pour conséquences une pollution plus grave, un prix de revient social plus élevé.

Le contrôle des déversements d'eaux usées est aussi très important. S'il n'y a pas de taxe, d'amende ni de contrôle des déversements d'eaux usées, l'entreprise n'aura pas de raison de diminuer la pollution. Maintenant en Chine, la taxe et les amendes sur le déversement d'eaux usées sont très bas ; il faut beaucoup les augmenter pour diminuer la pollution par les entreprises. Le contrôle de la pollution des eaux par les petites entreprises communales et rurales est une tâche très pénible. A courte échéance, il est nécessaire de fermer les petites entreprises trop polluantes, mais à long terme, le moyen idéal est le traitement unitaire des eaux usées industrielles et urbaines.

Il manque également une planification de la gestion des eaux. La plupart de villes en Chine n'ont pas une approche de la gestion intégrée concernant l'approvisionnement en eau, la gestion de la demande, et le contrôle de la pollution. L'investissement en installations d'approvisionnement en eau est basé généralement sur la quantité dont on a besoin, sans tenir compte de l'effet du prix de l'eau sur la consommation. Les villes et les régions doivent appliquer la nouvelle méthode de gestion intégrée du bassin versant pour établir leurs plans.

Augmenter l'efficacité de l'irrigation est une stratégie prioritaire de la gestion de la demande en eau agricole; cela peut être réalisé par la généralisation de la technologie d'irrigation rationnelle aidée par le gouvernement.

\section{Exemple 1: L'utilisation de l'eau par les industriels est plus efficace dans la province de Shanxi.}

Les secteurs industriels de la province de Shanxi ont fait beaucoup d'efforts pour la protection des ressources en eau. En 1980, la valeur moyenne d'eau produite pour l'usage d'eau industriel était de $22 \mathrm{yuan} / \mathrm{m}^{3}$; en 1990 , elle était de $48 \mathrm{yuan} / \mathrm{m}^{3}$; en 1995 , elle monte à $75 \mathrm{yuan} / \mathrm{m}^{3}$. En 1991 , le taux de réutilisation de l'eau dans le cycle industriel avait déjà dépassé la valeur fixée par l'Etat pour 2000, en 1993 ; ce taux est de $84,3 \%$. Selon l'analyse du processus de production, la plupart des investissements industriels de ces dernières années ont porté sur l'augmentation de la valeur de la ressource en eau. En même temps, l'approvisionnement en eau est devenu un frein au développement des secteurs industriels. Selon les statistiques, même si l'eau ne manque pas, la valeur de la production industrielle totale en 1993 sera de 65 milliards yuan (en augmentation de $22 \%$ ). A cause de l'insuffisance de l'approvisionnement en eau, quelques usines sont obligées d'arrêter la production pendant quelque temps. Si ce problème peut être résolu, la production industrielle pourra augmenter beaucoup plus grâce aux ressources en matières premières et aux conditions géographiques. Selon les estimations, s'il y a transfert d'eau d'une autre région, la valeur de la production industrielle de la province de Shanxi pourra augmenter de $75 \%$ de 1993 à 2000 , sinon, elle restera au niveau de production actuel.

Exemple 2: Traitement des eaux usées à Dalian : prix de revient du traitement égal au prix de revient des eaux courantes

Dalian est l'une des villes côtières qui manquent d'eau en Chine. Près de 40 à $50 \%$ des eaux courantes sont utilisées pour la production industrielle. Pendant l'étiage, quelques grands consommateurs de l'eau industriels sont obligés d'arrêter la production pendant 60 jours. En 1992, on a construit une station de traitement des eaux usées (qui traite $100000 \mathrm{~m}^{3}$ par jour). Les eaux usées traitées (elles atteignent la classe 4 de la qualité d'eau) fournissent aux entreprises non seulement des eaux moins chères, mais aussi augmentent la production industrielle tout en diminuant la pollution de l'eau.

La comparaison du prix de revient de la production industrielle en utilisant l'eau propre ou l'eau traitée est la suivante :

- Prix de l'eau courante : $\quad 1,5$ yuan/t

- Prix de revient de l'approvisionnement
en eau propre
1,2 yuan/t
- Taxe de la pollution
0,1 yuan/t
- Prix de l'eau usée traitée
0,8 yuan/t
- Prix de revient du traitement : 0,5 yuan/t

\subsection{Augmenter les investissements et garantir l'approvisionnement en eau}

C'est en augmentant les investissements que l'on pourra garantir l'approvisionnement en eau et améliorer la qualité de l'eau. Pour résoudre le problème de l'insuffisance de l'approvisionnement en eau du nord de la Chine, il faut investir pour les travaux de transfert d'eau du sud au nord. Mais ce travail ne doit pas remplacer les investissements pour la protection, le traitement des eaux usées et le recyclage de l'eau. Toutes les villes doivent investir pour renforcer l'approvisionnement en eau et les services d'assainissement. Ces investissements peuvent réduire la perte économique due à l'insuffisance d'approvisionnement en eau et aussi abaisser le prix de revient social de la pollution de l'eau. Le gouvernement ne peut pas supporter seul tous les investissements. Etablir une politique de prix rationnelle et des règles de gestion de l'environnement peut encourager et attirer les investissements privés dans ce domaine.

Toutes les villes doivent faire un gros effort pour collecter les eaux usées domestiques et industrielles et construire des stations d'épuration. Les investissements de collecte et traitement des eaux usées peuvent se réaliser en émettant des emprunts ou en attirant les investissements privés ou publics. 
Les villes de la Grande-Bretagne ou de France ont ellesmêmes recours à des emprunts. Dans quelques années, les entreprises auront les ressources financières suffisantes pour continuer les investissements. D'autre part, les projets d'approvisionnement en eau ou d'épuration peuvent attirer les investissements d'entreprises privées.

Le gouvernement chinois exige que toutes les grandes et moyennes villes aient des traitements primaires pour toutes les eaux usées n'atteignant pas le critère de la qualité pour l'irrigation. Il exige aussi des villes de donner des facilités pour installer des ouvrages de traitement et de collecte des eaux usées. Une bonne gestion de l'approvisionnement en eau peut abaisser les investissements pour l'approvisionnement et laisser des fonds pour la collecte et le traitement des eaux usées urbaines.

\subsection{Unification et coordination des organismes de gestion des eaux et de contrôle de la pollution}

Il faut penser globalement, mais agir aussi localement. Ainsi, la réforme des organismes de protection de l'eau en Chine doit être basée sur les bassins versants dans le but d'unifier la gestion des eaux et le contrôle de la pollution. Cela demande d'établir des organismes puissants de gestion du bassin versant.

\subsubsection{Rôle des comités de la gestion du bassin versant}

Les sept comités des bassins versants doivent fonctionner comme des entreprises, avec des autorités et des responsabilités législatives pour leur propre bassin versant :

- Captage des eaux,

- Lutte contre les inondations,

- Drainage,

- Navigation,

- Débits à la sortie des usines hydroélectriques,

- Contrôle des débits,

- Protection de la qualité de l'eau de toutes les rivières et des affluents,

- Contrôle de la pollution côtière.

Les comités des bassins versants doivent avoir le droit d'établir des critères de prix de l'eau dans leur bassin versant de façon à garantir les dépenses d'exploitation et des nouveaux projets hydrauliques pour le contrôle des débits de leur bassin versant. Une partie des revenus du contrôle de la pollution et des captages est distribuée à l'administration municipale et aux entreprises pour le traitement des eaux usées.

Le comité du bassin versant doit établir un système de l'inspection de la qualité de l'eau, réaliser des inspections journalière, hebdomadaire et mensuelle sur les grandes rivières et leurs affluents. Les bureaux de la protection de l'environnement d'Etat et locaux doivent établir un réseau de surveillance indépendant pour vérifier les données de l'inspection du bassin versant.

Les villes et les communes doivent unifier la collecte et le traitement des eaux usées domestiques et industrielles. Désormais, il est interdit de déverser les eaux usées directement dans les rivières naturelles. Les eaux usées doivent être collectées par des égouts urbains puis traitées. Du point de vue économique, le prix de revient du traitement global (domestique et industriel) peut être moindre que la somme des traitements séparés. Les eaux usées industrielles doivent être cependant prétraitées (pour éliminer les matières toxiques et les métaux lourds) avant d'entrer dans les réseaux d'égouts urbains. Il faut établir, annoncer et mettre en œuvre le plus tôt possible le régime de taxe sur les déversements des eaux usées. La taxe sur les eaux usées industrielles déversées dans les réseaux d'égouts urbains doit tenir compte des paramètres suivants :

- Le prix du traitement primaire et secondaire ;

- La quantité totale de DCO et DBO5 ;

- Le prix de revient d'élimination des matières en suspension et des boues ;

- Le taux de toxicité.

La collecte et le traitement des eaux usées doivent être pris en charge par des organismes de l'administration municipale, et ces organismes doivent fonctionner comme entreprises financièrement indépendantes. Le prix de l'eau doit tenir compte des taxes sur les eaux usées, ces taxes vont être distribuées aux secteurs de la collecte et du traitement des eaux usées.

\subsubsection{Rôle des bureaux de la protection de l'environne- ment d'Etat et locaux}

- L'autorité des bureaux de protection de l'environnement d'Etat et locaux doit être élargie à la gestion et l'exécution de tous les critères de protection de l'environnement.

- Compléter et améliorer la gestion par des lois : établir les critères d'approvisionnement en eau, de collecte des eaux usées, de qualité de l'eau de rivières, et de contrôle de la pollution.

- Vérifier et approuver les règles de protection de la nature, des écosystèmes et des activités de loisirs.

- Garantir le fonctionnement des entreprises d'approvisionnement en eau et de traitement des eaux usées suivant les critères établis. Si les entreprises ne respectent pas ces critères, le bureau de la protection de l'environnement a le droit de les pénaliser.

- Inviter les experts à évaluer la qualité de l'eau des rivières et des côtes.

- Surveiller le traitement des déchets, surtout le traitement des déchets toxiques.

- Faire des recommandations pour le contrôle de la pollution à chaque échelon du gouvernement.

- Examiner, vérifier et contrôler les processus de la production qui sont susceptibles de polluer et de nuire à la santé publique

\subsubsection{Rôle du ministère des Eaux}

- Surveiller les aménagements hydrauliques des bassins versants.

- Examiner et ratifier tous les critères du prix de la production et de distribution de l'eau.

- Avoir autorité pour toutes les formes de gestion des rivières y compris l'évacuation de crue de grands barrages hydroélectriques.

- Etablir des zones de protection, garantir la quantité minimum d'approvisionnement en eau de ces zones.

\subsubsection{Rôle du ministère de la Construction}

- Surveiller la technologie d'approvisionnement en eau et de collecte des eaux usées des entreprises.

- Enquêter sur tous les accidents pouvant influencer la qualité de l'eau.

— Fournir la consultation technique sur la politique de l'eau potable. 
- Trouver des causes potentiellement nuisibles à la qualité de l'eau, et y déployer la recherche scientifique.

- Donner la directive pour les moyens d'analyse et d'inspection de la qualité de l'eau potable.

- Examiner tous les grands projets de l'approvisionnement en eau et évaluer l'impact des produits chimiques et autres matières.

\subsubsection{Rôle du public}

La gestion des eaux et le contrôle de la pollution sont une affaire d'intérêt public ; elle demande les interventions et le soutien de la population. L'intervention publique dans l'établissement des lois de gestion des eaux peut améliorer l'efficacité et les effets de la gestion des eaux.

\section{Réflexions}

1. Il ne faut pas laisser la population penser que quand on a payé sa facture et la taxe, on a le droit de gaspiller l'eau.

2. Le réseau unitaire de traitement des eaux usées urbaines ne peut pas être utilisé par toutes les villes ; il faut choisir selon la situation un réseau unitaire, séparatif ou mixte.

\section{D CONCLUSION}

Les investissements insuffisants et la surcharge des systèmes de ressource de l'eau ont déjà provoqué de graves nuisances. Les inondations sont devenues de plus en plus fréquentes et destructives. En 1994, la perte due aux inonda- tions était estimée à près de 160 milliards de yuan ; en 1996, cette perte est estimée à près de 220 milliards de yuan. Sur plus de 660 villes en Chine, plus de 300 sont susceptibles de manquer d'eau. Chaque année, il manque plus de 6 milliards de $\mathrm{m}^{3}$ d'eau en Chine et la production industrielle en a été diminuée de près de 120 milliards de yuan. Les fleuves, les lacs et les réservoirs ont subi des pollutions plus ou moins grandes, à part quelques rivières intérieures et grands réservoirs. Cette pollution tend à s'aggraver. Ces problèmes sont plus évidents dans les rivières proches des villes industrielles. La perte due à la pollution est maintenant estimée à 33 milliards de yuan par an (NEPA, 1997).

A côté des inondations, le manque d'eau et la pollution sont considérés comme les deux principaux facteurs en rapport aux eaux nuisibles au développement économique et social de la Chine. Pour résoudre ces deux problèmes, il faut beaucoup d'investissements, une politique efficace de gestion des eaux et des organismes compétents de gestion.

N. B. : Mme Dang Welhong, qui a traduit cet article, est une fidèle lectrice de la Houille Blanche depuis qu'elle travaille à I'Institut de recherche des Informations du ministère des Eaux de la Chine. Elle a obtenu beaucoup d'informations sur la technique des eaux en France et dans d'autres pays grâce à la revue et elle souhaiterait que les ingénieurs français et des autres pays puissent également connaître la situation des eaux de la Chine et aider la Chine à développer la technique dans le domaine de l'eau. Ici, elle nous a présenté la situation des eaux de la Chine. Comme la pénurie et la pollution de l'eau en Chine seront de plus en plus graves dans le proche avenir, la Chine devrait constituer un grand marché de l'eau pour les investisseurs étrangers. 